\title{
Dentipellis tasmanica sp. nov. (Hericiaceae, Basidiomycota) from Australia
}

\author{
Xiao-Hong Ji', Qian Chen', Genevieve Gates ${ }^{2}$, Ping $\mathrm{Du}^{3}$ \\ I Institute of Microbiology, PO Box 61, Beijing Forestry University, Beijing 100083, China 2 Tasmanian \\ Institute of Agriculture, Private Bag 54, Hobart, Tasmania 7001, Australia 3 College of Life Science and \\ Technology, Yangtze Normal University, Chongqing 408100, China
}

Corresponding authors: Xiao-HongJi (xhji2010@163.com); Ping Du (duping7374@163.com)

Academic editor: Zai-Wei Ge | Received 19 July 2018 | Accepted 18 September 2018 | Published 11 October 2018

Citation: Ji X-H, Chen Q, Gates G, Du P (2018) Dentipellis tasmanica sp. nov. (Hericiaceae, Basidiomycota) from Australia. MycoKeys 41: 29-38. https://doi.org/10.3897/mycokeys.41.28485

\begin{abstract}
Dentipellis tasmanica sp. nov. is described and illustrated from Tasmania, Australia based on rDNA evidence and morphological characters. It is characterised by an annual growth habit; resupinate basidiocarps up to $100 \mathrm{~cm}$ long; spines cream when fresh and cinnamon when dry, up to $3 \mathrm{~mm}$ long and a few glued at tips when dry; distinct white fibrillous to cottony margin; a monomitic hyphal structure with non-amyloid, non-dextrinoid and cyanophilous generative hyphae; the presence of gloeoplerous hyphae and gloeocystidia which become dark blue in Melzer's reagent; the presence of chlamydospores in the subiculum and rough basidiospores measuring 3.5-4.5 × 2.4-3.2 $\mu \mathrm{m}$. A molecular study based on the combined ITS (internal transcribed spacer region) and 28 S (the large nuclear ribosomal RNA subunit) dataset supports the new species in Dentipellis. A key to species of Dentipellis sensu stricto is provided.
\end{abstract}

\section{Keywords}

hydnoid fungi; Russulales; taxonomy; wood-inhabiting fungi

\section{Introduction}

Dentipellis Donk, typified by D. fragilis (Pers.) Donk, is a hydnaceous genus in the Russulales and is characterised by a wood-inhabiting resupinate fruiting body with soft spines, a monomitic hyphal structure with clamp connections on the generative hyphae and amyloid, rough basidiospores (Ginns 1986, Dai et al. 2009, Zhou and Dai 2013). Zhou and Dai (2013) demonstrated that Dentipellis was polyphyletic and segregated

Copyright Xiao-Hong Ji et al. This is an open access article distributed under the terms of the Creative Commons Attribution License (CC BY 4.0), which permits unrestricted use, distribution, and reproduction in any medium, provided the original author and source are credited. 
Dentipellis leptodon (Mont.) Maas Geest. and Dentipellis taiwaniana Sheng H. Wu from Dentipellis to a new genus of Dentipellicula Y.C. Dai \& L.W. Zhou based on ITS and 28S rDNA sequences. Besides, Dentipellopsis Y.C. Dai \& L.W. Zhou is erected as a new genus and characters are provided in a generic key to distinguish Dentipellicula, Dentipellis and Dentipellopsis that morphologically are highly similar, as well as a key to the current species in Dentipellis (Zhou and Dai 2013). Recently, based on molecular and morphological analyses, more new taxa were described in Dentipellis sensu lato (Zhou and Dai 2013, Chen et al. 2015, Shen and Wang 2017, Yuan et al. 2018) and, indeed, all Dentipellis spp. were found from the northern Hemisphere (Ginns 1986, Dai et al. 2009, Zhou and Dai 2013, Shen and Wang 2017, Yuan et al. 2018).

During a field trip to Tasmania, the island state of Australia, three wood-inhabiting specimens with soft spines were collected and, based on the morphological characters, they belong to Dentipellis. After phylogenetic analysis of ITS and $28 \mathrm{~S}$ sequences and examination of the morphology in the laboratory, they turn out to represent a new species. This is so far the first species of Dentipellis found in the southern Hemisphere. In this paper, we present an illustrated description and an identification key to accepted species of Dentipellis worldwide.

\section{Materials and methods}

\section{Morphological studies}

Thin sections were studied microscopically according to Chen et al. (2016) at magnifications $\leq 1000 \times$ using a Nikon Eclipse $80 \mathrm{i}$ microscope with phase contrast illumination. Drawings were made with the aid of a drawing tube. Microscopic features, measurements and drawings were made from sections stained with Cotton Blue and Melzer's reagent. Spores were measured from sections cut from the tubes. To present spore size variation, the $5 \%$ of measurements excluded from each end of the range are given in parentheses. Basidiospore apiculus lengths were not included in the measurements.

Abbreviations include:

\begin{tabular}{|c|c|c|c|}
\hline $\begin{array}{l}\text { IKI } \\
\text { IKI- }\end{array}$ & $\begin{array}{l}\text { Melzer's reagent, } \\
\text { negative in Melzer's reagent, }\end{array}$ & $\mathrm{L}$ & $\begin{array}{l}\text { mean spore length (arithmetic } \\
\text { average of all spores), }\end{array}$ \\
\hline $\mathrm{KI}+$ & amyloid in Melzer's reagent, & W & $\begin{array}{l}\text { mean spore width (arithmetic } \\
\text { average of all spores) }\end{array}$ \\
\hline & Cotton Blue, & $\mathrm{Q}$ & the L/W ratio, \\
\hline B & $\begin{array}{l}\text { cyanophilous, } \\
\text { acyanophilous, }\end{array}$ & $\mathrm{n}$ & $\begin{array}{l}\text { number of spores measured from } \\
\text { the given number of specimens. }\end{array}$ \\
\hline
\end{tabular}

Colour terms follow Petersen (1996). The studied specimens are deposited in the herbaria as cited below; herbarium abbreviations follow Thiers (2014). 


\section{Molecular study and phylogenetic analysis}

A CTAB rapid plant genome extraction kit (Aidlab Biotechnologies, Beijing) was used to obtain PCR products from dried specimens, according to the manufacturer's instructions with some modifications (Wu et al. 2017). The primer pair ITS4 and ITS5 was used for amplification of the ITS region (White et al. 1990), while the primer pair LR0R and LR7 (http://www.biology.duke.edu/fungi/mycolab/primers. $\mathrm{htm}$ ) was used for providing the D1-D4 regions of the 28S (https://unite.ut.ee/primers.php). The PCR procedure for ITS was as follows: initial denaturation at $95^{\circ} \mathrm{C}$ for $3 \mathrm{~min}$, followed by 35 cycles at $94^{\circ} \mathrm{C}$ for $40 \mathrm{~s}, 54^{\circ} \mathrm{C}$ for $45 \mathrm{~s}$ and $72{ }^{\circ} \mathrm{C}$ for $1 \mathrm{~min}$, with a final extension of $72{ }^{\circ} \mathrm{C}$ for $10 \mathrm{~min}$. The PCR procedure for $28 \mathrm{~S}$ was as follows: initial denaturation at $94^{\circ} \mathrm{C}$ for $1 \mathrm{~min}$, followed by 35 cycles at $94^{\circ} \mathrm{C}$ for $30 \mathrm{~s}$, $50{ }^{\circ} \mathrm{C}$ for $1 \mathrm{~min}$ and $72{ }^{\circ} \mathrm{C}$ for $1.5 \mathrm{~min}$, with a final extension of $72{ }^{\circ} \mathrm{C}$ for $10 \mathrm{~min}$. The PCR products were purified and sequenced in Beijing Genomics Institute, China with the same primers.

New sequences, deposited in GenBank (Table 1), were aligned with additional sequences retrieved from GenBank (Table 1) using BioEdit 7.0.5.3 (Hall 1999) and ClustalX 1.83 (Chenna et al. 2003). Bondarzewia podocarpi Y.C. Dai \& B.K. Cui and B. occidentalis Jia J. Chen, B.K. Cui \& Y.C. Dai were chosen as outgroups, consulting Dai et al. (2010) and Zhou and Dai (2013). Prior to phylogenetic analysis, ambiguous regions at the start and the end of the alignment were deleted and gaps were manually adjusted to optimise the alignment. The edited alignment was deposited at TreeBase (submission ID 22975; www.treebase.org).

The method of phylogenetic analysis followed Chen et al. (2016). Maximum parsimony (MP) analysis was performed in PAUP* version 4.0b10 (Swofford 2002). All characters were equally weighted and gaps were treated as missing data. Trees were inferred using the heuristic search option with tree-bisection reconnection (TBR) branch swapping and 1,000 random sequence additions. Max-trees were set to 5,000, branches of zero length were collapsed and all parsimonious trees were saved. Clade robustness was assessed using a bootstrap (BT) analysis with 1,000 replicates (Felsenstein 1985). Phylogenetic trees were visualised using Treeview (Page 1996).

MrModeltest 2.3 (Posada and Crandall 1998, Nylander 2004) was used to determine the best-fit evolution model of the combined dataset for Bayesian Inference (BI). BI was calculated with MrBayes 3.1.2 (Ronquist and Huelsenbeck 2003) with a general time reversible (GTR) model of DNA substitution and an invgamma distribution rate variation across sites. Four Markov chains were performed for 2 runs from random starting trees for 500,000 generations of the combined ITS and 28S dataset and trees were sampled every 100 generations. The burn-in was set to discard the first $25 \%$ of the trees. A majority rule consensus tree of all remaining trees was calculated. Nodes that received BT support $\geq 50 \%$ and Bayesian posterior probabilities (BPP) $\geq 0.95$ were considered as significantly supported. 
Table I. Specimens and GenBank accession number of sequences used in this study.

\begin{tabular}{|c|c|c|c|c|}
\hline \multirow{2}{*}{ SPECIES } & \multirow{2}{*}{ SAMPLE NO. } & \multirow{2}{*}{ LOCALITY } & \multicolumn{2}{|c|}{ GENBANK ACCESSION NO. } \\
\hline & & & ITS & nLSU \\
\hline Bondarzewia occidentalis & DAOM F-415 & Canada & DQ200923 & DQ234539 \\
\hline B. podocarpi & Dai 9261 & China & KJ583207 & KJ583221 \\
\hline Dentipellicula austroafricana & Dai 12580 & South Africa & KJ855274 & KJ855275 \\
\hline D. leptodon & GB 011123 & Uganda & EU118625 & EU118625 \\
\hline \multirow{2}{*}{ D. taiwaniana } & Dai 10867 & China & JQ349115 & JQ349101 \\
\hline & Cui 8346 & China & JQ349114 & JQ349100 \\
\hline \multirow{2}{*}{ Dentipellis coniferarum } & Cui 10063 & China & JQ349106 & JQ349092 \\
\hline & Yuan 5623 & China & JQ349107 & JQ349093 \\
\hline D. dissita & NH 6280 & Canada & AF506386 & AF506386 \\
\hline \multirow{2}{*}{ D. fragilis } & Dai 12550 & China & JQ349110 & JQ349096 \\
\hline & Dai 9009 & China & JQ349108 & JQ349094 \\
\hline \multirow{2}{*}{ D. longiuscula } & He 20120717-5 & China & KR108235 & KR108238 \\
\hline & He 20120717-7 & China & KR108234 & KR108239 \\
\hline D. microspora & Cui 10035 & China & JQ349112 & JQ349098 \\
\hline \multirow{3}{*}{ D. rhizomorpha } & Dai 17474 & China & MG020134 & MG020137 \\
\hline & Dai 17477 & China & MG020135 & MG020138 \\
\hline & Dai 17481 & China & MG020136 & MG020139 \\
\hline \multirow{3}{*}{ D. tasmanica } & Dai 18737 & China & MH571698 $^{a}$ & MH571701 $^{\mathrm{a}}$ \\
\hline & Dai 18767 & China & MH571699a & MH571702 \\
\hline & Dai 18768 & China & MH571700 & MH571703 \\
\hline \multirow{2}{*}{ D. tropicalis } & Cui 8545 & China & KR108236 & KR108240 \\
\hline & He 1993 & China & KR108237 & KR108241 \\
\hline Dentipellopsis dacrydicola & Dai 12004 & China & JQ349104 & JQ349089 \\
\hline D. dacrydicola & Dai 12010 & China & - & JQ349090 \\
\hline Hericium abietis & $\mathrm{NH} 6990$ & Canada & AF506456 & AF506456 \\
\hline H. alpestre & NH 13240 & Russia & AF506457 & AF506457 \\
\hline H. americanum & DAOM F-21467 & Canada & AF506458 & AF506458 \\
\hline H. coralloides & NH 282 & Sweden & AF506459 & AF506459 \\
\hline H. erinaceus & NH 12163 & Russia & AF506460 & AF506460 \\
\hline Laxitextum bicolor & NH 5166 & Sweden & AF310102 & AF310102 \\
\hline Pseudowrightoporia japonica & Dai 7221 & China & FJ644289 & KM107882 \\
\hline Wrightoporiopsis biennis & Cui 8457 & China & KJ807066 & KJ807074 \\
\hline
\end{tabular}

a Sequences newly generated in this study; the new species is shown in bold.

\section{Results}

The combined ITS and 28S dataset included sequences from 31 fungal collections representing 22 species. The dataset had an aligned length of 1792 characters, of which 1218 characters are constant, 126 are variable and parsimony-uninformative and 448 (37\%) are parsimony-informative. MP analysis yielded 2 equally parsimonious trees $(\mathrm{TL}=$ $1343, \mathrm{CI}=0.653, \mathrm{RI}=0.793, \mathrm{RC}=0.518, \mathrm{HI}=0.347)$. The best-fit model for the combined ITS and $28 \mathrm{~S}$ sequences dataset estimated and applied in the Bayesian analysis: $\mathrm{GTR}+\mathrm{I}+\mathrm{G}$, lset $n s t=6$, rates $=$ invgamma; prset statefreqpr $=$ dirichlet $(1,1,1,1) . \mathrm{BI}$ 
resulted in a similar topology with an average standard deviation of split frequencies = 0.006203 to MP analysis and, thus, only the MP tree was provided. Both BT values $(\geq 50 \%)$ and BPPs $(\geq 0.95)$ are shown at the nodes (Fig. 1$)$.

Three sampled specimens of the new species, Dentipellis tasmanica, formed a wellsupported lineage (100\% MP and 1 BPPs), indicating they are phylogenetically distinct from other species (Fig. 1).

\section{Taxonomy}

Dentipellis tasmanica Y.C. Dai, G.M. Gates, X.H. Ji \& P. Du, sp. nov. MycoBank: MB 827073

Figs 1, 2

Diagnosis. Differs from other Dentipellis species by its gloeoplerous hyphae and gloeocystidia that become dark blue in Melzer's reagent and the presence of chlamydospores in subiculum.

Holotype. AUSTRALIA. Tasmania: Arve River Streamside Reserve, $43^{\circ} 10^{\prime}$ S, $146^{\circ} 48.5^{\prime}$ E, elev. 160 m, on fallen trunk of Nothofagus sp., 15 May 2018, Dai 18767 (M, isotype in BJFC; ITS GenBank accession number: MH571699, 28S GenBank accession number: $\mathrm{MH} 571702)$.

Etymology. Tasmanica (Lat.): referring to the species collected from Tasmania of Australia.

Basidiomata. Annual, resupinate, inseparable from substratum, soft corky, without odour or taste when fresh, fragile upon drying, up to $100 \mathrm{~cm}$ long, $40 \mathrm{~cm}$ wide and $3.5 \mathrm{~mm}$ thick at centre. Hymenophore with spines, cream when fresh and cinnamon when dry, spines up to $3 \mathrm{~mm}$ long, 2-3 per $\mathrm{mm}$ across base, soft corky to fragile, a few glued at tips when dry; margin distinct, white, fibrillous to cottony, up to $5 \mathrm{~mm}$ wide; spines, cream, becoming fragile and clay-buff when dry, up to $3 \mathrm{~mm}$ long. Subiculum very thin, soft corky, white to cream, $<1 \mathrm{~mm}$ thick.

Hyphal structure. Hyphal system monomitic; generative hyphae with clamp connections, IKI-, $\mathrm{CB}+$; the colour and size unchanged in $\mathrm{KOH}$.

Subiculum. Generative hyphae colourless, thin- to slightly thick-walled, frequently branched, flexuous, interwoven, $3-4.5 \mu \mathrm{m}$ in diam. Gloeoplerous hyphae occasionally present, dark blue in Melzer's reagent. Chlamydospores present, ellipsoid, thickwalled, $5-5.6 \times 2.8-3.3 \mu \mathrm{m}$.

Hymenophoral trama. Generative hyphae colourless, thin-walled, frequently branched, straight, parallel along the spines, $2.8-4 \mu \mathrm{m}$ in diam. Gloeocystidia abundant, colourless, thin- to slightly thick-walled, clavate, contents oily and dark blue in Melzer's reagent, rooting deep from the trama, up to a few hundred microns long, the cystidia-like apical part 30-45 × 5-8 $\mu \mathrm{m}$. Oily material abundant amongst trama.

Hymenium. Cystidioles colorless, thin-walled, ventricose with elongated apical portion, bearing some irregular crystals, $30-45 \times 5-8 \mu \mathrm{m}$; basidia clavate with four 


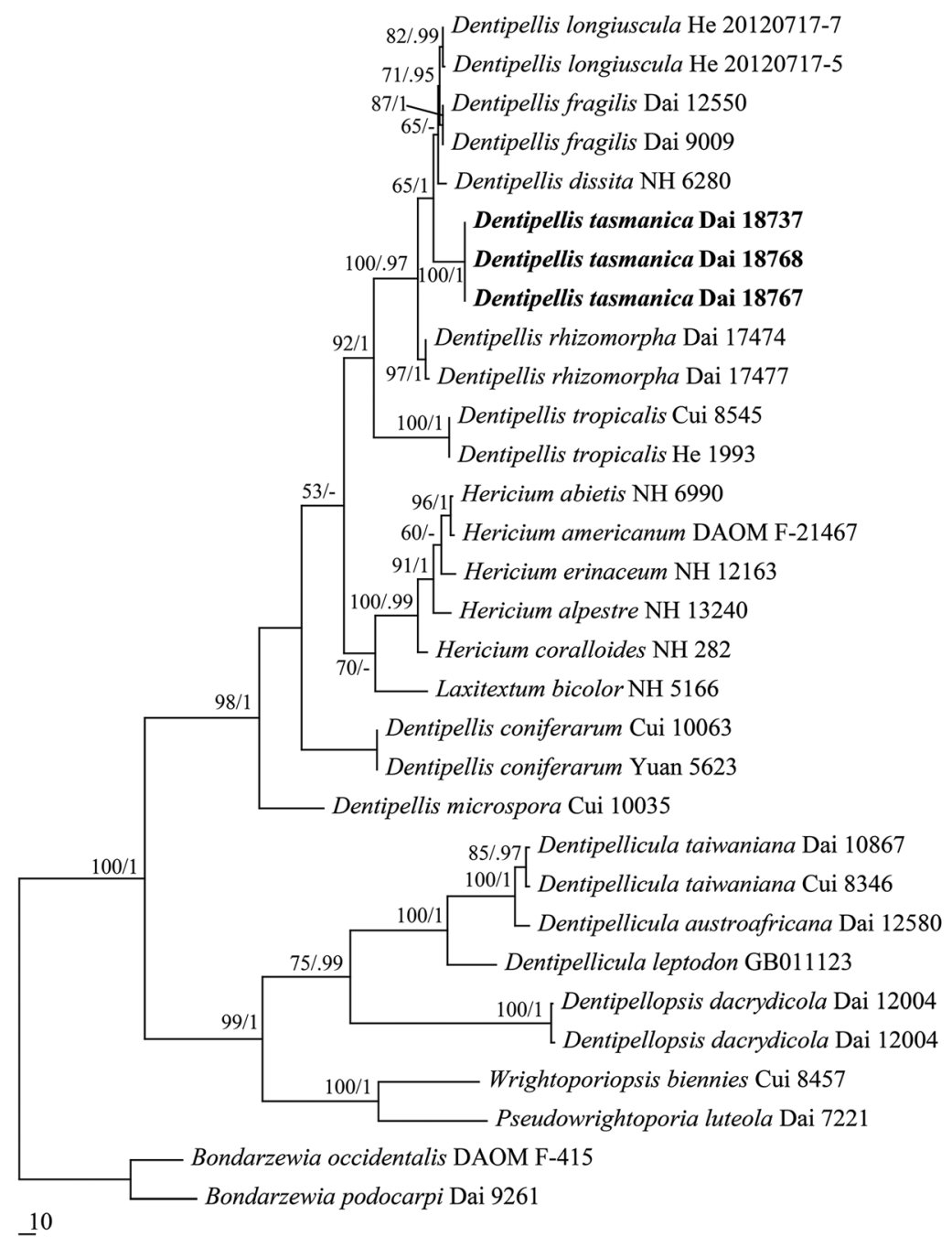

Figure I. Strict consensus tree illustrating the phylogenetic position of Dentipellis tasmanica, generated by the maximum parsimony method based on ITS $+28 S$ sequence data. Branches are labelled with parsimony bootstrap values $\geq 50 \%$ and Bayesian posterior probabilities $\geq 0.95$. Bondarzewia podocarpi and B. occidentalis are used to root the tree. Branch lengths reflect expected changes per site as indicated by the scale.

sterigmata and a basal clamp connection, 20-26 × 3-4.5 $\mu \mathrm{m}$. Basidiospores ellipsoid, coloruless, thin-walled, densely echinulate, IKI+, CB+, (3.4-)3.5-4.5(-4.8) $\times 2.4-$ 3.2(-3.5) $\mu \mathrm{m}, \mathrm{L}=3.99 \mu \mathrm{m}, \mathrm{W}=2.92 \mu \mathrm{m}, \mathrm{Q}=1.36-1.39(\mathrm{n}=90 / 3)$.

Additional specimens examined (paratypes). AUSTRALIA. Tasmania: Arve River Streamside Reserve, on fallen trunk of Nothofagus sp., 15 May 2018, Dai 18768 (M, duplicate in BJFC; ITS GenBank accession number: MH571700, 28S GenBank accession 


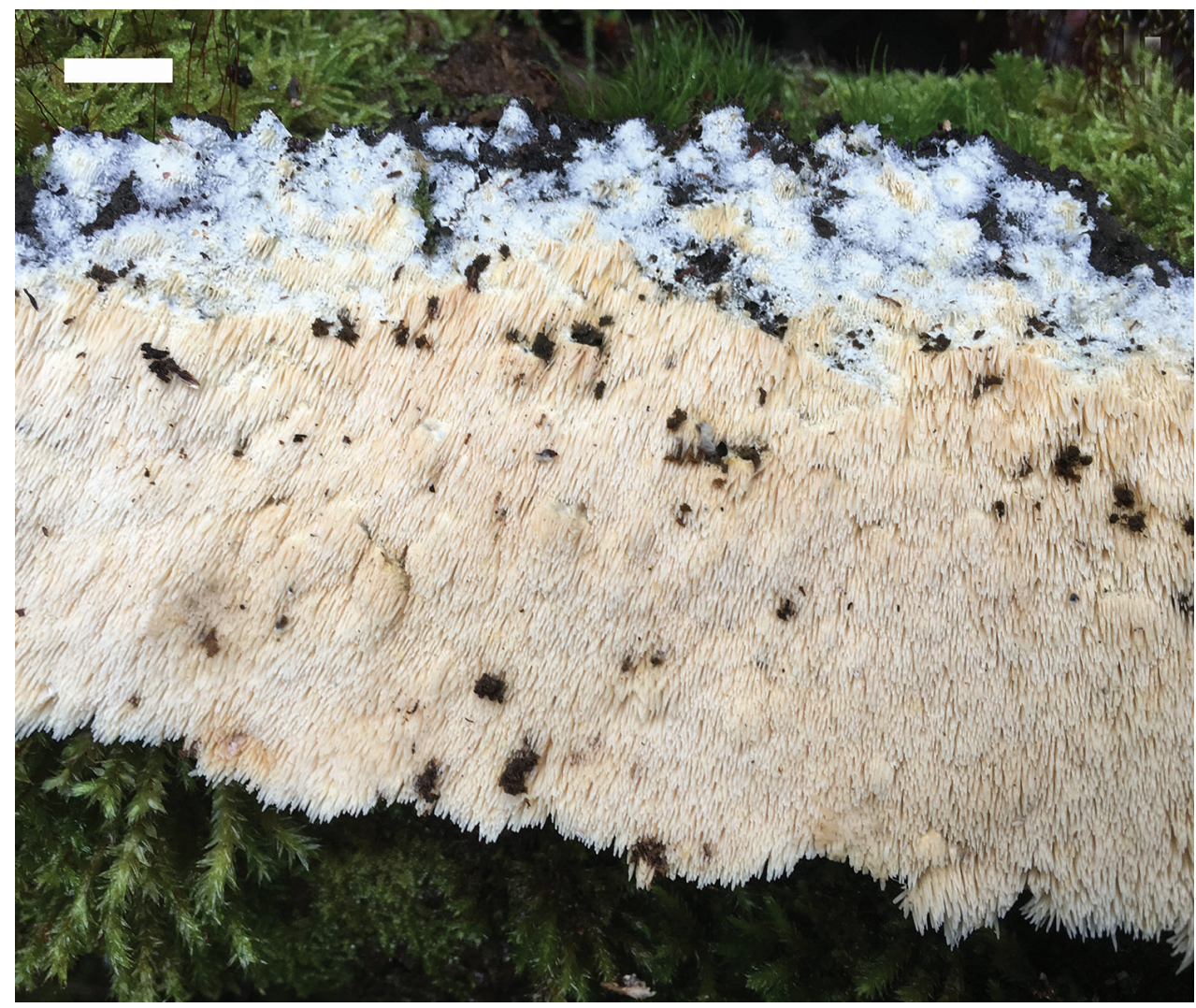

Figure 2. A fresh basidiocarp of Dentipellis tasmanica (holotype). Scale bar: $1 \mathrm{~cm}$.

number: MH571703); Mt Field National Park, 4241'S, 146²2'E, elev., 180 m, on fallen trunk of Nothofagus sp., 14 May 2018, Dai 18737 (M, duplicate in BJFC; ITS GenBank accession number: MH571698, 28S GenBank accession number: MH571701).

\section{Discussion}

Morphologically, Dentipellis tasmanica is characterised by spines, cream when fresh; distinct white fibrillous to cottony margin; a monomitic hyphal structure with generative hyphae bearing clamp connections; the presence of gloeoplerous hyphae and gloeocystidia which become dark blue in Melzer's reagent and presence of chlamydospores in the subiculum. Phylogenetically, three samples of D. tasmanica formed a distinct lineage with strong support (100\% MP, 1.0 BPPs) and are distant from other taxa (Fig. 1). Both morphology and rDNA sequence data confirmed that D. tasmanica is a new species in Dentipellis.

Dentipellis tasmanica was considered as Dentipellicula leptodon (Mont.) Y.C. Dai \& L.W. Zhou (Gates and Ratkowsky 2016) as having similar basidiospores (3.5-4.5 × 2.4-3.3 $\mu \mathrm{m}$ 

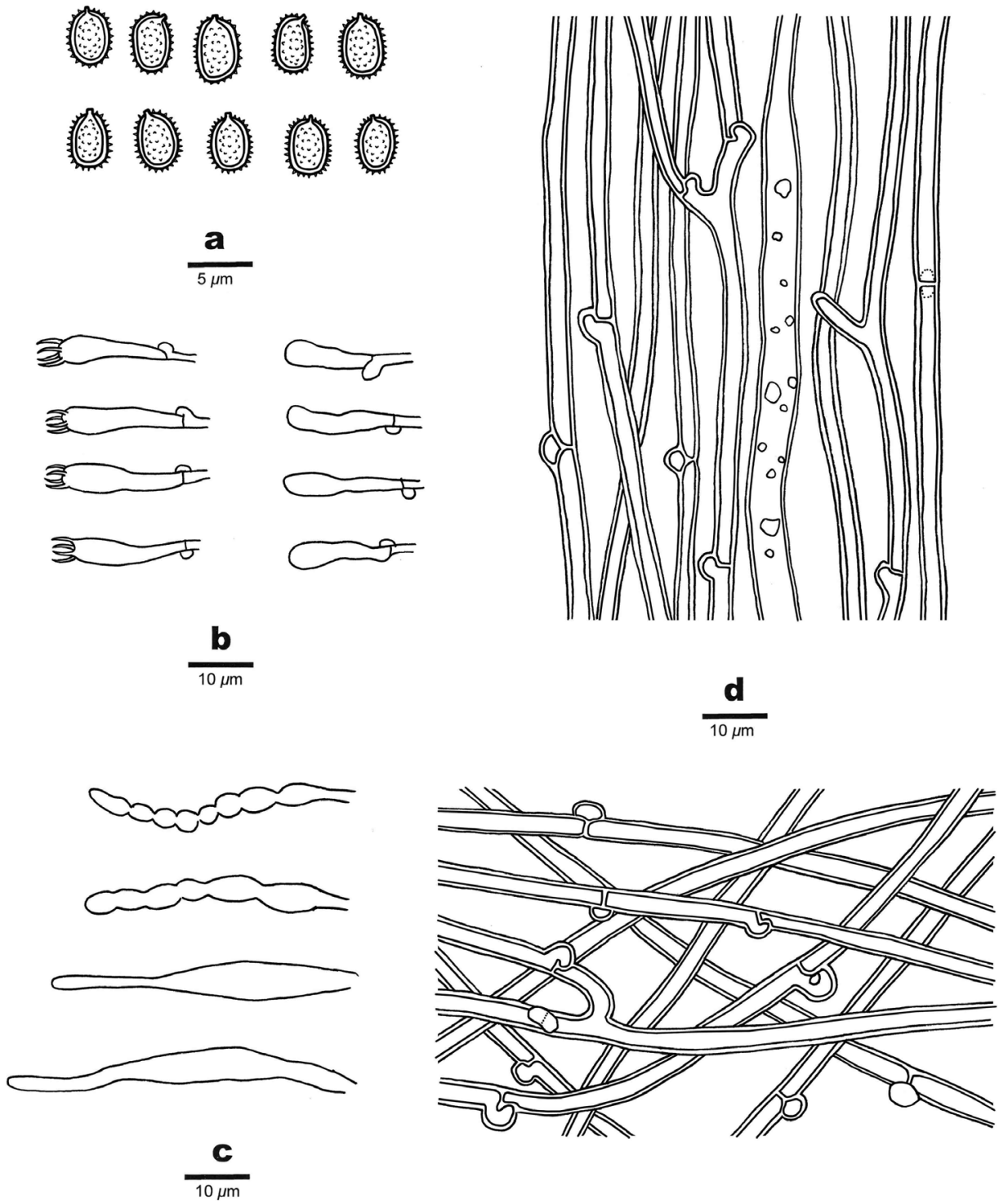

$\frac{\mathbf{e}}{10 \mu \mathrm{m}}$

Figure 3. Microscopic structures of Dentipellis tasmanica (holotype). a Basidiospores b Basidia and basidioles c Gloeocystidia and Cystidioles $\mathbf{d}$ Hyphae from trama e Hyphae from subiculum.

vs. 3.2-4 $1 \times 2.4-3 \mu \mathrm{m}$, Ginns 1986 ), but gloeocystidia and gloeoplerous hyphae in $D$. leptodon are yellowish in Melzer's reagent and it lacks chlamydospores in subiculum.

Phylogenetically, Dentipellis tasmanica is more closely related to D. rhizomorpha Yuan \& Y.C. Dai, D. fragilis, D. dissita and D. longiuscula (Fig.1). However, D. rhizomorpha has denser spines (5-7 per mm vs. 2-3 per $\mathrm{mm}$ in $D$. tasmanica), lacks gloeoplerous hyphae 
and gloeocystidia. $D$. fragilis and $D$. dissita differ from $D$. tasmanica in having larger basidiospores $(5-5.8 \times 4.1-4.9 \mu \mathrm{m}$ in D. fragilis, 4.2-4.7 × 3.2-3.7 $\mu \mathrm{m}$ in D. dissita; Dai et al. 2009). D. longiuscula is distinguished from $D$. tasmanica by lacking gloeoplerous hyphae and gloeocystidia and having larger basidiospores (5-6 ×3-3.6 $\mu \mathrm{m}$; Shen and Wang 2017).

\section{Key to species of Dentipellis}

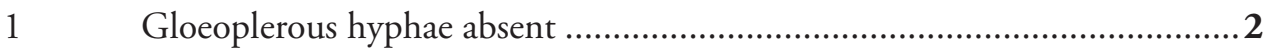

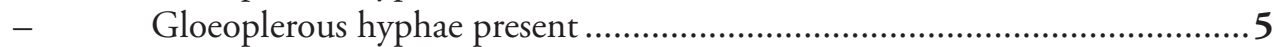

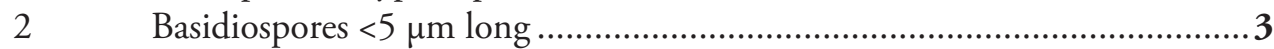

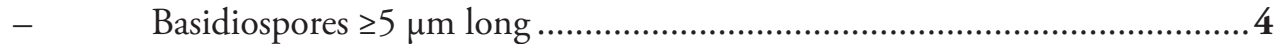

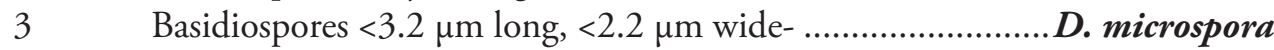

- $\quad$ Basidiospores $>3.2 \mu \mathrm{m}$ long, $>2.2 \mu \mathrm{m}$ wide- ...................... D. rhizomorpha

$4 \quad$ Gloeocystidia absent ............................................................ longiuscula

- Gloeocystidia present ............................................................... D. tropicalis

$5 \quad$ Basidiocarps becoming brown when bruised ........................ D. coniferarum

- Basidiocarps unchanged when bruised ..................................................6

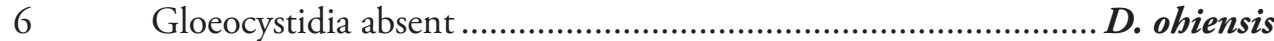

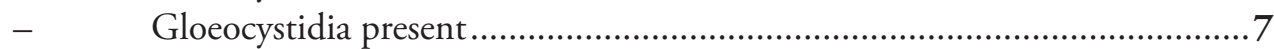

$7 \quad$ Gloeocystidia dark blue in IKI, basidiospores $<3.2 \mu \mathrm{m}$ wide... D. tasmanica

- $\quad$ Gloeocystidia yellowish in IKI, basidiospores $>3.2 \mu$ m wide .......................8

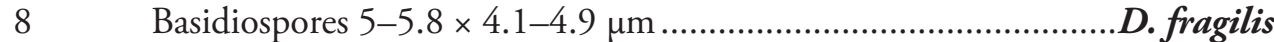

- $\quad$ Basidiospores 4.2-4.7 × 3.2-3.7 $\mu \mathrm{m}$........................................ D. dissita

\section{Acknowledgements}

We would like to express our deep thanks to Prof. Yu-Cheng Dai (Beijing Forestry University) who allowed us to study his specimens. The research is supported by the National Natural Science Foundation of China (Project No. 31750001).

\section{References}

Chen JJ, Cui BK, Dai YC (2016) Global diversity and molecular systematics of Wrightoporia s. 1. (Russulales, Basidiomycota). Persoonia 37: 21-36. https://doi. org/10.3767/003158516X689666

Chen JJ, Cui BK, He SH, Cooper JA, Barrett MD, Chen JL, Dai YC (2016) Molecular phylogeny and global diversity of the remarkable genus Bondarzewia (Basidiomycota, Russulales). Mycologia 108: 697-708. https://doi.org/10.3852/14-216

Chen JJ, Shen LL, Dai YC (2015) Dentipellicula austroafricana sp. nov. supported by morphological and phylogenetic analyses. Mycotaxon 130: 17-25. https://doi.org/10.5248/130.17 
Chenna R, Sugawara H, Koike T, Lopez R, Gibson TJ, Higgins DG, Thompson JD (2003) Multiple sequence alignment with the Clustal series of programs. Nucleic Acids Research 31: 3497-3500. https://doi.org/10.1093/nar/gkg500

Dai YC, Cui BK, Liu XY (2010) Bondarzewia podocarpi, a new and remarkable polypore from tropical China. Mycologia 102: 881-886. https://doi.org/10.3852/09-050

Dai YC, Xiong HY, Wu SH (2009) Notes on Dentipellis (Russulales, Basidiomycota). Mycosystema 28: 668-671.

Felsenstein J (1985) Confidence intervals on phylogenetics: an approach using bootstrap. Evolution 39: 783-791. https://doi.org/10.2307/2408678

Gates G, Ratkowsky D (2016) A field guide to Tasmanian fungi. Tasmanian Field Naturalists Club, Hobart, 1-249.

Ginns J (1986) The genus Dentipellis (Hericiaceae). Windahlia 16: 35-45.

Hall TA (1999) Bioedit: a user-friendly biological sequence alignment editor and analysis program for Windows 95/98/NT. Nucleic Acids Symp Ser 41: 95-98

Nylander JAA (2004) MrModeltest v2. Program distributed by the author. Evolutionary Biology Centre, Uppsala University.

Page RMD (1996) Treeview: An application to display phylogenetic trees on personal computers. Comput Appl Biosci 12: 357-358.

Petersen JH (1996) The Danish Mycological Society's color-chart. Foreningen til Svampekundskabens Fremme, Greve, 1-6.

Posada D, Crandall KA (1998) Modeltest: testing the model of DNA substitution. Bioinformatics 14: 817-818. https://doi.org/10.1093/bioinformatics/14.9.817

Ronquist F, Huelsenbeck JP (2003) MRBAYES 3: Bayesian phylogenetic inference under mixed models. Bioinformatics 19: 1572-1574. https://doi.org/10.1093/bioinformatics/btg180

Shen LL, Wang M (2017) Morphological characteristics and molecular data reveal two new species of Dentipellis from China. Phytotaxa 323: 69. https://doi.org/10.11646/phytotaxa.323.1.5

Swofford DL (2002) PAUP*: Phylogenetic analysis using parsimony (*and other methods). Version 4.0b10. Sinauer Associates, Sunderland, Massachusetts.

Thiers B (2014) Index Herbariorum: A global directory of public herbaria and associated staff. New York Botanical Garden's Virtual Herbarium.

White TJ, Bruns TD, Lee S, Taylor J (1990) Amplification and direct sequencing of fungal ribosomal RNA genes for phylogenetics. In: Innis MA, Gelfand DH, Sninsky JJ, White TJ (Eds) PCR protocols, a guide to methods and applications. Academic, San Diego, 315322. https://doi.org/10.1016/B978-0-12-372180-8.50042-1

Wu, F, Chen JJ, Ji XH, Vlasák J, Dai YC (2017) Phylogeny and diversity of the morphologically similar polypore genera Rigidoporus, Physisporinus, Oxyporus and Leucophellinus. Mycologia 109: 749-765. https://doi.org/10.1080/00275514.2017.1405215

Yuan Y, Ren GJ, Dai YC (2018) Dentipellis rhizomorpha sp. nov. supported by morphological and phylogenetic analyses. Nova Hedwigia 107: 131-140. https://doi.org/10.1127/ nova_hedwigia/2018/0459

Zhou LW, Dai YC (2013) Taxonomy and phylogeny of hydnoid Russulales: two new genera, three new species and two new combination species. Mycologia 105: 636-649. https://doi. org/10.3852/12-011 